\title{
Scintillation, OSL and TSL Properties of Yttria Stabilized Zirconia Crystal
}

\author{
Daisuke Nakauchi, Go Okada, Takayuki Yanagida
}

\author{
Graduate School of Materials Science, Nara Institute of Science and Technology (NAIST), \\ 8916-5 Takayama-cho, Ikoma, Nara 630-0192, Japan \\ nakauchi.daisuke.mv7@ms.naist.jp
}

\begin{abstract}
We investigated the scintillation and dosimeter properties of yttria stabilized zirconia ( $\mathrm{YSZ}, \mathrm{Y}_{2} \mathrm{O}_{3^{-}}$ $\mathrm{ZrO}_{2}$ ) crystal. It showed $70-80 \%$ in-line transmittance at wavelength longer than $300 \mathrm{~nm}$. Under excitations around the bandgap energy, several intense photoluminescence (PL) emission peaks appeared at 450, 550, 570 and $600 \mathrm{~nm}$, and the PL decay time constants were 5.5 and $20.7 \mathrm{~ns}$. In the X-ray induced scintillation spectrum, an intense emission peak around $450 \mathrm{~nm}$ was observed, and the scintillation decay time constants were 14.4 and $169.8 \mathrm{~ns}$. After irradiations with X-rays, thermally stimulated luminescence (TSL) glow peaks appeared at 100, 200 and $450{ }^{\circ} \mathrm{C}$. Also, after X-ray irradiations, optically stimulated luminescence (OSL) was detected around $500 \mathrm{~nm}$ with $650 \mathrm{~nm}$ stimulation.
\end{abstract}

\section{Introduction}

In order to measure invisible ionizing radiations, luminescent materials have commonly been utilized in radiation detectors. Such materials may be classified into two types - scintillator and dosimeter. The former is a phosphor which converts ionizing radiation to thousands of visible photons immediately. Scintillators are used in various fields of radiation detections such as medical imaging [1], security system [2], well-logging [3], high energy physics [4] and astrophysics [5]. On the other hand, dosimeters are mainly used for personal dose monitoring. Such materials store and accumulate the absorbed radiation energy, and the accumulated energy is released in a form of light emission during heating (thermally stimulated luminescence: TSL) or optical stimulation (optically stimulated luminescence: OSL). The emission intensity is proportional to the dose accumulated, hence the dose can be indirectly measured.

In this study, we have studied the scintillation, TSL and OSL properties of yttria stabilized zirconia (YSZ, $\mathrm{Y}_{2} \mathrm{O}_{3}-\mathrm{ZrO}_{2}$ ) crystal. $\mathrm{YSZ}$ is, in general, well-known as a solid state ionic conductor which has a high mechanical strength and chemical stability. With these notable characteristics, YSZ has been used in various applications including gas sensors [6], conductive ceramics [7], thermal barrier coatings [8], and solid fuel cells [9]. In these applications, understanding the behavior of YSZ under excitation is important. The emission by the recombination of the photo-excited electrons with holes 
is called the photoluminescence (PL), and the PL measurements often give us information towards understanding the nature of electrons and holes in materials. Thus, the PL properties of YSZ show its electronic band structure and indispensable defects in synthesis of bulk forms. To date, the PL properties of non-doped and rare earth doped YSZ have been reported elsewhere [10-16], and YSZ is well known as one of the good phosphors, which has various emission wavelengths. However, as far as we are aware, no studies have been reported on the radiation induced luminescence properties of YSZ. YSZ is an interesting material since it has a high density of $6 \mathrm{~g} / \mathrm{cm}^{3}$ so the radiation stopping power is high and radiation energy is effectively absorbed. Also, in some host materials, an inclusion of zirconium would enhances the detection probability of the double beta decay $(0 v \beta \beta)$ which tests the validity of the grand unification theory [17], and yittria itself acts as a very bright scintillator [18, 19]. Moreover, in addition to the scintillation properties, it is of a great importance to comprehensively investigate the dosimeter properties together since these are complementarily related as reported in [20]. In other words, for example, a bright scintillator material is not a sensitive dosimeter and vice versa. Therefore, such comprehensive study helps reveal a room to improve the performance as a radiation detector.

\section{Experimental procedures}

The YSZ crystal sample used in this study is a commercial product and was purchased from NEOTRON, Japan. Fig. 1 shows an appearance of the sample. The sample size was $10 \times 10 \times 1 \mathrm{~mm}^{3}$. The wide surfaces were polished for optical characterizations, and it was visually colorless. The inline transmittance was evaluated by using JASCO V670 spectrometer in the spectral range from 190 to $2700 \mathrm{~nm}$ with $1 \mathrm{~nm}$ step. The PL emission spectra under various excitation wavelengths were measured by using a spectrofluorometer (FP-8600, JASCO). The PL decay time profile monitoring at $450 \mathrm{~nm}$ under $280 \mathrm{~nm}$ excitation was evaluated by using Hamamatsu Quantaurus- $\tau$ (Hamamatsu Photonics). The reason why we used the $280 \mathrm{~nm}$ excitation was because it was the shortest and most efficient excitation wavelength available in the instrument.

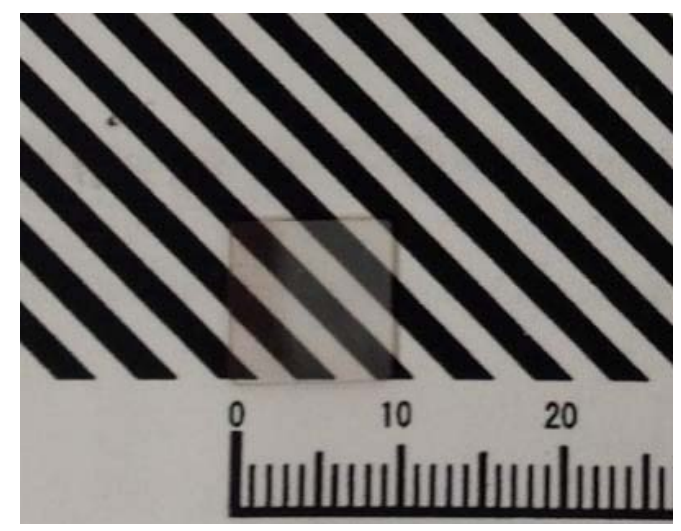

Fig. 1 Picture of YSZ crystal with colorless and transparent. 
X-ray induced radioluminescence (RL) spectrum was measured by utilizing our original setup [21]. The irradiation source was X-ray generator equipped with a tungsten anode target (XRB80P\&N200X4550, Spellman). During the measurements, the X-ray generator was supplied with the voltage of $80 \mathrm{kV}$ and tube current of $2.5 \mathrm{~mA}$. While the sample was irradiated by X-rays, the scintillation emissions from the sample were fed into the spectrometer through a $2 \mathrm{~m}$ optical fiber to measure the scintillation spectrum. The spectrometer (Andor DU-420-BU2 CCD and Shamrock 163 monochromator) was cooled down to $193 \mathrm{~K}$ by a Peltier module to reduce the thermal noise. Further, we have measured the scintillation decay time and afterglow profiles using a pulsed X-ray source equipped afterglow characterization system [22]. In order to characterize relatively shallow trapping centers induced by X-ray irradiations, the TSL glow curves were measured by TL-2000 (Nanogray, Japan) with the heating rate of $1{ }^{\circ} \mathrm{C} / \mathrm{s}$ over the temperature range from 50 to $490{ }^{\circ} \mathrm{C}$ [23]. On the other hand, for deeper trapping centers, OSL was also studied under $650 \mathrm{~nm}$ stimulation by using FP8600 spectrofluorometer (JASCO). Except the TSL, all the other measurements mentioned above were performed at room temperature unless specified. 


\section{Results and Discussion}

The in-line transmittance spectrum of YSZ crystal is shown in Fig. 2. The transmittance was 70$80 \%$ at the wavelengths longer than $300 \mathrm{~nm}$, but a rapid edge was clearly observed around $300 \mathrm{~nm}$ due to the bandgap absorption. The observed data was consistent with the bandgap energy $(5.0 \mathrm{eV}=250$ $\mathrm{nm}$ ) of YSZ [24]. No particular absorption band structures were observed in the wavelength range of our measurement.

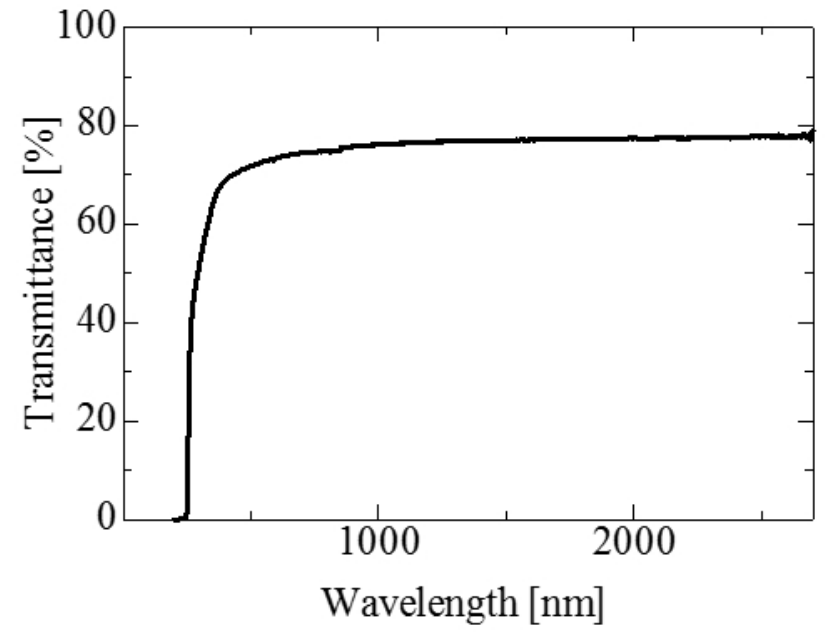

Fig. 2 In-line transmittance of YSZ crystal.

The PL emission spectra are shown in Fig. 3. Under excitations with 305, 210 and $250 \mathrm{~nm}$, one intense emission band around $450 \mathrm{~nm}$, one emission band around $550 \mathrm{~nm}$ and two emission bands around 570 and $600 \mathrm{~nm}$ appeared, respectively. N. G. Petrik et al. reported that YSZ has three main defect structures with oxygen vacancies, and these affect to the PL spectrum [12]. That is, the emission at $450 \mathrm{~nm}$ is due to a defect structure where all the nearest neighbors of $\mathrm{Zr}^{4+}$ are oxygen vacancy, and the emissions at 550 and $600 \mathrm{~nm}$ correspond to defect structures where a single oxygen vacancy has one and two neighboring $\mathrm{Y}^{3+}$ ions, respectively. 


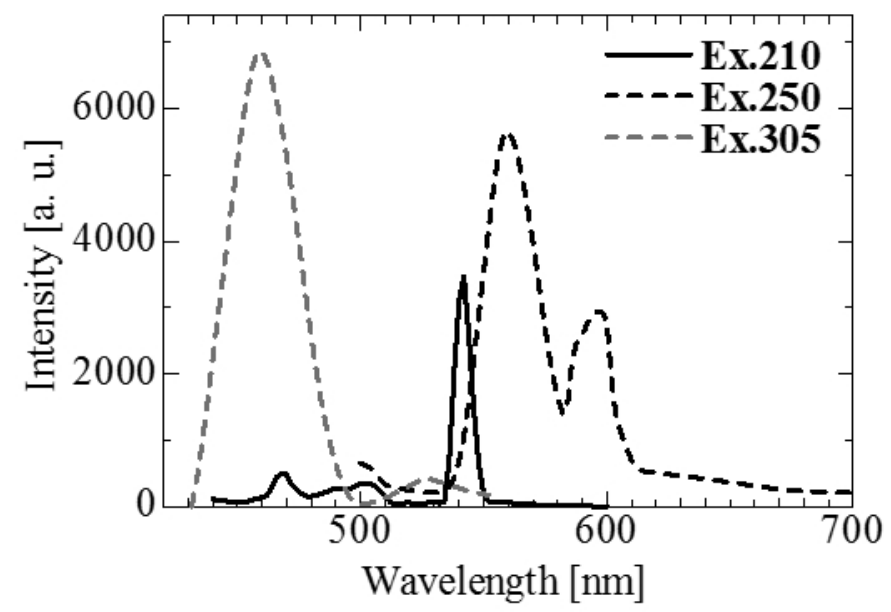

Fig 3. PL emission spectra upon various excitation wavelengths.

The PL decay time profiles of YSZ monitoring at $450 \mathrm{~nm}$ emission band under $280 \mathrm{~nm}$ excitation is illustrated in Fig. 4. The obtained decay time constants are 1.1, 5.5 and 20.7 ns. We think that the fastest component $(1.1 \mathrm{~ns})$ would be due to the instrumental response because the timing resolution of the instrument was around $1 \mathrm{~ns}$. The $5.5 \mathrm{~ns}$ component is caused by some kinds of defects of $\mathrm{ZrO}_{2}$ because similar results were reported previously [25]. The origin of the $20.7 \mathrm{~ns}$ component is under investigation but a similar decay profile with $20 \mathrm{~ns}$ of lifetime was previously reported by another research [26]. In this report, it was mentioned that the lifetime of this decay component varies by heat treatment in air; therefore, the origin of this $20 \mathrm{~ns}$ component would be due to the oxygen vacancy.

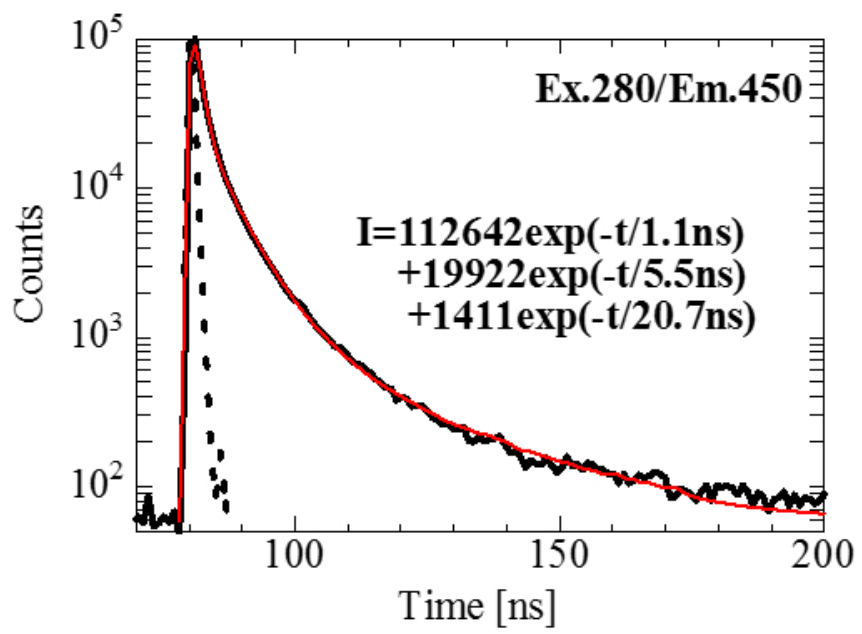

Fig. 4. PL decay time profiles of YSZ monitoring at $450 \mathrm{~nm}$ under 280 excitation.

The X-ray induced scintillation spectrum of YSZ is shown in Fig. 5. As it was observed in the PL spectra, an emission band was observed around $450 \mathrm{~nm}$. Although no reports can be found about scintillation properties of both $\mathrm{YSZ}$ and $\mathrm{ZrO}_{2}$, a previous work [18] reported that $\mathrm{Y}_{2} \mathrm{O}_{3}$ ceramics show 
scintillation with a broad emission peaking around $350 \mathrm{~nm}$, but the emission peak doses not agree with our result. Therefore, we think that the scintillation observed around $450 \mathrm{~nm}$ is mainly due to the defect centers surrounded by $\mathrm{Zr}^{4+}$ ions as suggested by the PL measurements. The broad structure of the emission suggest that there are some other emissions with different origins, but these are overlapped each other to have such a broad structure. Additionally, self-trapped excitons (STE) might cause the broad peak since STE is observed in $\mathrm{Y}_{2} \mathrm{O}_{3}$ [18] and other simple oxides. These minor emission may be due to the other defects observed in our PL measurements above. In addition, we think that a weak sharp peak appeared around $610 \mathrm{~nm}$ would be due to an unexpected rare earth contamination in the synthesis process since the $\mathrm{Y}^{3+}$ site is suitable for rare earth ions to substitute, and many rare earth ions tend to show sharp line emissions regardless of the host crystal fields due to the inter $4 \mathrm{f}$ shell transitions.

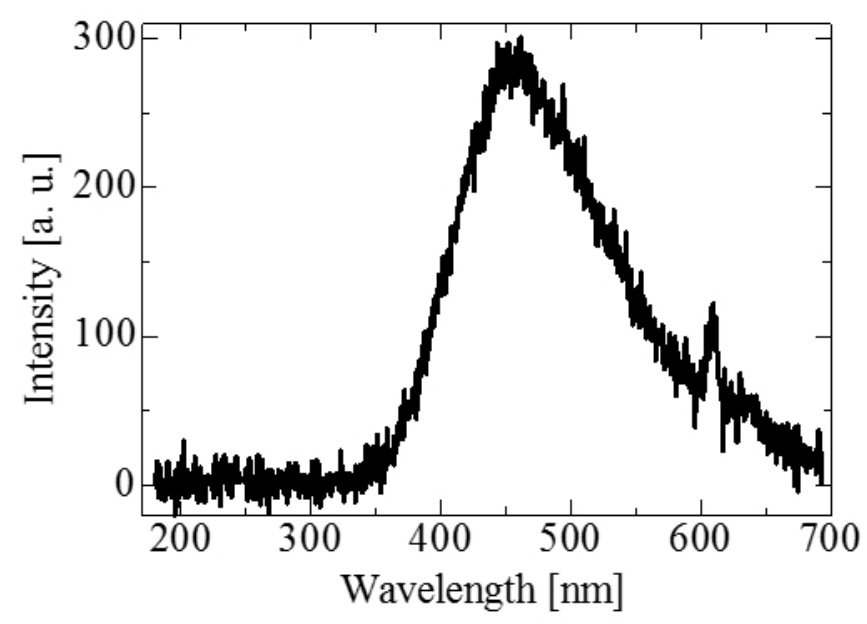

Fig. 5. X-ray induced scintillation spectrum of YSZ.

Fig. 6 shows the X-ray excited scintillation decay time profile. It can be decomposed into three exponential decay functions, and the deducted decay time constants were 1.3, 14.4 and $169.8 \mathrm{~ns}$. The fastest component is from the instrument since the response time of the PMT (R7400, Hamamatsu) was around $1 \mathrm{~ns}$. The origin of the $14.4 \mathrm{~ns}$ component would be ascribed to the oxygen vacancy because the PL decay time ( $\sim 20 \mathrm{~ns})$ of the oxygen vacancy was similar value. The origin of the slowest component is unclear but one possibility was the STE. In $\mathrm{Y}_{2} \mathrm{O}_{3}$ ceramic which showed a very broad band scintillation from 300 to $500 \mathrm{~nm}$ due to the STE, a similar scintillation decay time of $107 \mathrm{~ns}$ was reported [18]. 


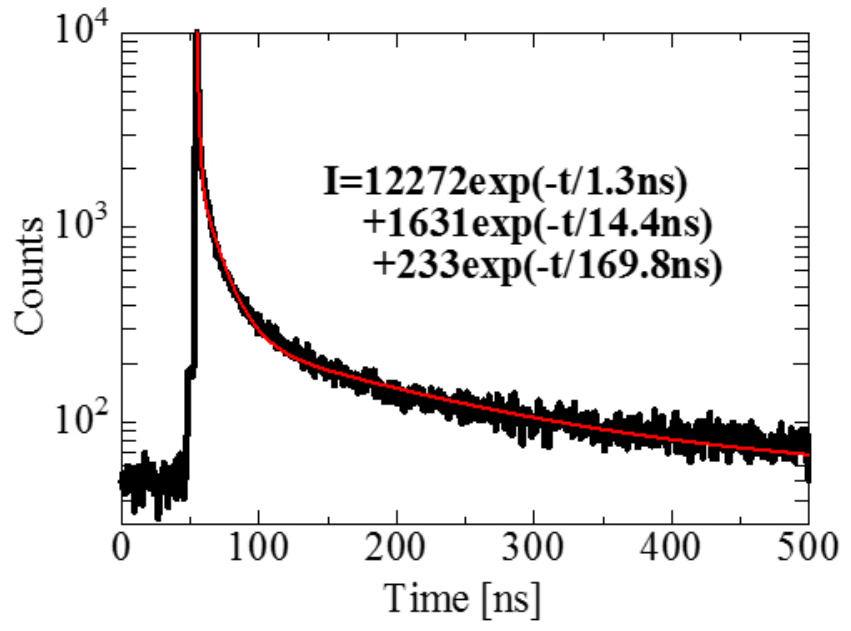

Fig. 6. Scintillation decay time profile of YSZ under pulse X-ray excitation.

The other important timing property for scintillation detector, the X-ray induced afterglow profile of YSZ is shown in Fig. 7. This is a transient luminescence intensity profile over a longer time range after longer irradiation time in contrast with the ordinary scintillation decay profile illustrated in Fig. 6. Here, the irradiation time length was $2 \mathrm{~ms}$, which is the simulated value assuming that, in a scanning X-ray imaging setup, a line detector with the width of $1 \mathrm{~mm}$ takes an X-ray image of an object travelling at the speed of $50 \mathrm{~cm} / \mathrm{s}$ on a line belt. The afterglow level at $20 \mathrm{~ms}$ after the irradiation was stopped was $0.06 \%$ of the peak intensity. This value is much bigger than common scintillators such as $\mathrm{CdWO}_{4}$ and $\mathrm{BGO}[22]$. Such high afterglow level suggests that there are a large number of shallow traps so some of the X-ray generated charges are temporarily captured at these traps before recombination to emit light. Detailed discussions of this process can be found elsewhere [27].

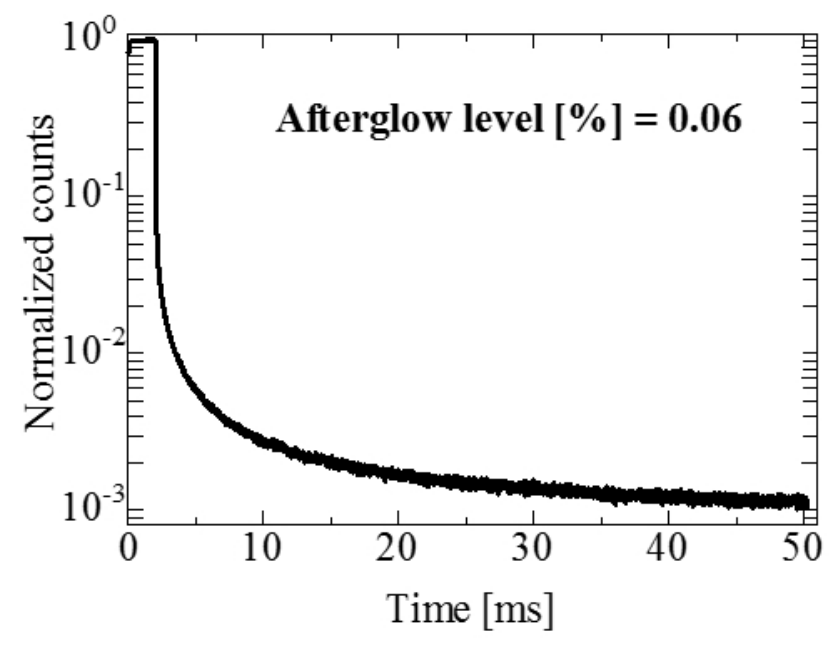

Fig. 7. Afterglow curve of YSZ after the X-ray irradiation was cut off. 
In order to further study the shallow charge traps and confirm the cause of large afterglow level observed in Fig. 7, we measured the TSL glow curve. The measured TSL glow curve is shown in Fig. 8. There were three glow peaks detected at 100,210 and $450{ }^{\circ} \mathrm{C}$. The 100 and $210{ }^{\circ} \mathrm{C}$ peaks were relatively weak while the $450{ }^{\circ} \mathrm{C}$ peak was strong. This complicated structure in the glow curve indicates that there are several different charge traps/defects, and this fact is consistent with our observation in PL spectra which also have very complicated emission spectral shape with various excitation wavelengths due to several different defects acting luminescent centers. It was also confirmed that the presence of the low temperature glow peak at $100{ }^{\circ} \mathrm{C}$ is the evidence of shallow trapping centers, and this must be the cause of the large afterglow level (Fig. 7). These two weak glow peaks at 100 and $210{ }^{\circ} \mathrm{C}$ were consistent with other experimental results observed in YSZ after X-ray or charged particles irradiation $[28,29]$. However, the strongest glow peak at $450{ }^{\circ} \mathrm{C}$ had not been reported. The activation energy against this glow peak was derived using the initial rise method [30], and the estimated value was $0.36 \mathrm{eV}$. In this analysis, we assumed that the rise part of the glow peak is simply approximated by $\ln I(T)=$ const $-\frac{E}{k T}$, where $I(T), E, k$; and $T$ represent the TSL intensity, activation energy, Boltzmann constant and temperature, respectively.

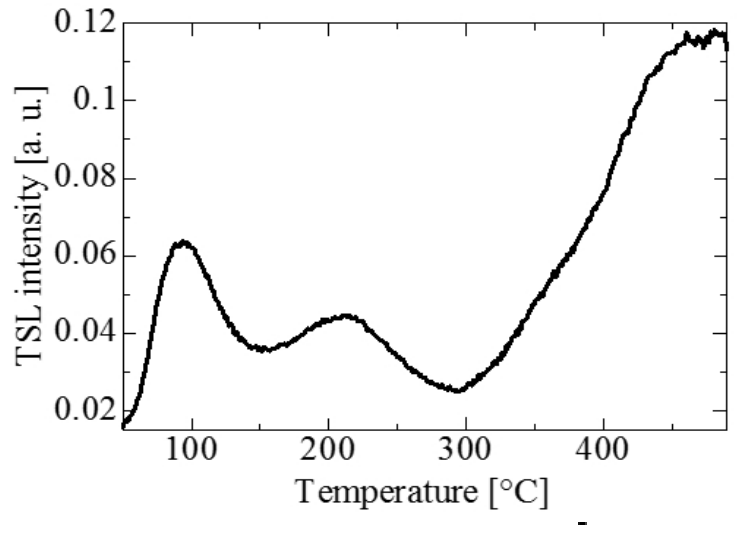

Fig. 8. TSL glow curve of YSZ.

Fig. 9 shows emission spectra while exposing the sample to the light at $650 \mathrm{~nm}$. Since this is a lowto-high energy photon conversion, one may think that this may be an up-conversion emission. When the sample was irradiated with the voltage of $40 \mathrm{kV}$ and tube current of $5.2 \mathrm{~mA}$ for 1 and $5 \mathrm{~min}$, it turned out that the emission intensity becomes stronger with longer X-ray irradiation performed prior to the measurements, though the differences are not so large. Moreover, as illustrate in Fig. 10, this emission intensity gradually decreases with the exposure time. This behavior may be explained as, in the up-conversion process, the cross-section between the excitation and emission bands, or in other words energy transfer efficiency, increases by X-ray irradiations due to the generation of defect centers 
etc. Another possibility would be that this emission is not up-conversion but OSL. It is a typical behavior of OSL to have larger intensity against the irradiation dose and fading during the exposure besides the fact that the emission is very stable in the present case.

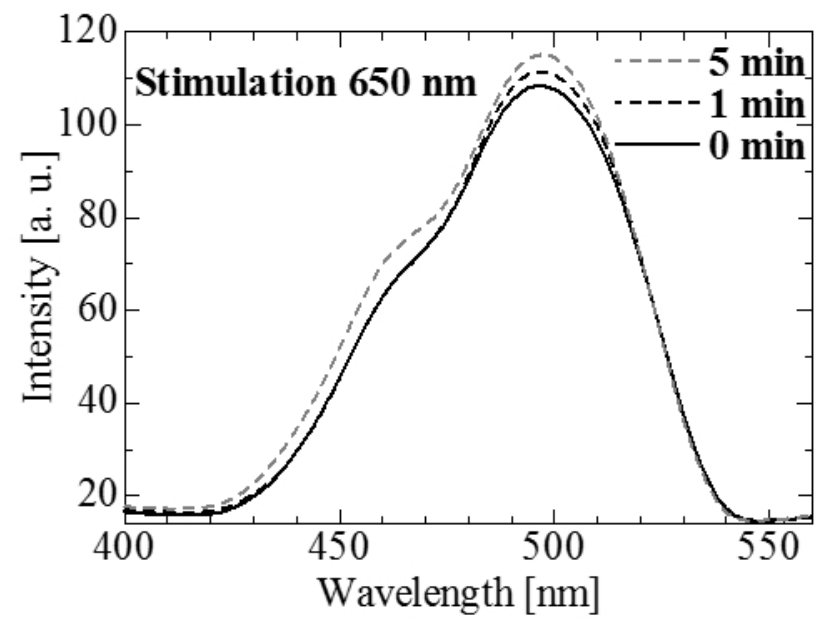

Fig. 9. OSL of YSZ spectrum upon $650 \mathrm{~nm}$ stimulation.

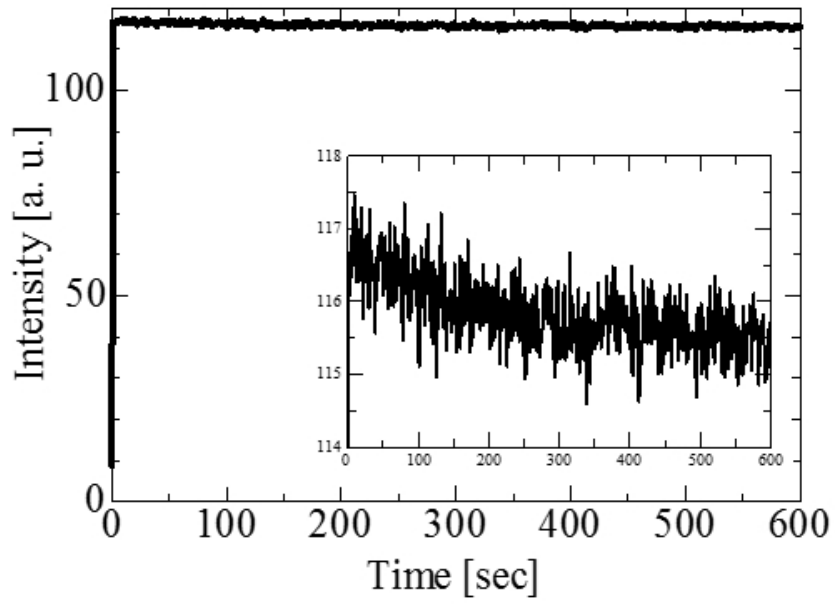

Fig. 10. Fading profile of OSL monitoring at $500 \mathrm{~nm}$ with $650 \mathrm{~nm}$ stimulation.

\section{Conclusion}

We investigated the optical, scintillation, TSL, and OSL properties of commercially available YSZ crystal. In the scintillation spectrum, an intense emission peak at $450 \mathrm{~nm}$ was observed, and the origin was mainly due to the oxygen vacancies surrounded by $\mathrm{Zr}^{4+}$ ions. The scintillation decay times of YSZ were relatively fast, 14.4 and $169.8 \mathrm{~ns}$. Having a fast scintillation is very advantageous for photon counting applications. YSZ showed TSL glow peaks at $100,200,450{ }^{\circ} \mathrm{C}$ and a stable OSL. It is confirmed that YSZ crystal can be potentially applicable for scintillator and dosimeter materials.

\section{Acknowledgement}


This work was supported by a Grant in Aid for Scientific Research (A)-26249147 from the Ministry of Education, Culture, Sports, Science and Technology of the Japanese government (MEXT) and partially by JST A-step. Green Photonics Research from MEXT and the Cooperative Research Project of Research Institute of Electronics, Shizuoka University, are also acknowledged.

\section{Reference}

[1] T. Yanagida, A. Yoshikawa, Y. Yokota, K. Kamada, Y. Usuki, S. Yamamoto, M. Miyake, M.

Baba, K. Sasaki, M. Ito, IEEE Trans. Nucl. Sci. 57 (2010) 14921495.

[2] D. Totsuka, T. Yanagida, K. Fukuda, N. Kawaguchi, Y. Fujimoto, Y. Yokota, A. Yoshikawa, Nucl. Instrum. Methods A 659 (2011) 399402.

[3] T. Yanagida, Y. Fujimoto, S. Kurosawa, K. Kamada, H. Takahashi, Y. Fukazawa, M. Nikl, V. Chani, Jpn. J. Appl. Phys. 52 (2013) 076401.

[4] T. Ito, M. Kokubun, T. Takashima, T. Yanagida, S. Hirakuri, R. Miyawaki, H. Takahashi, K. Makishima, T. Tanaka, K. Nakazawa, T. Takahashi, T. Honda, IEEE Trans. Nucl. Sci. 53 (2006) 29832990.

[5] K. Yamaoka, M. Ohno, Y. Terada, S. Hong, J. Kotoku, Y. Okada, A. Tsutsui, Y. Endo, K. Abe, Y. Fukazawa, S. Hirakuri, T. Hiruta, K. Itoh, T. Itoh, T. Kamae, M. Kawaharada, N. Kawano, K. Kawashima, T. Kishishita, T. Kitaguchi, M. Kokubun, G.M. Madejski, K. Makishima, T. Mitani, R. Miyawaki, T. Murakami, M.M. Murashima, K. Nakazawa, H. Niko, M. Nomachi, K. Oonuki, G. Sato, M. Suzuki, H. Takahashi, I. Takahashi, T. Takahashi, S. Takeda, K. Tamura, T. Tanaka, M. Tashiro, S. Watanabe, T. Yanagida, D. Yonetoku, IEEE Trans. Nucl. Sci. 52 (2005) 27652772.

[6] E.D. Bartolomeo, M.L. Grill, J.W. Yoon, E. Traversa, J. Am. Ceram. Soc. 87 (2004) 1883.

[7] M. Price, J. Dong, X. Gu, S.A. Speakman, E.A. Payzant, J. Am. Ceram. Soc. 88 (2005) 1812.

[8] F. Tarasi, M. Medraj, A. Dolatabadi, R.S. Lima, C. Moreau, J. Am. Ceram. Soc. 95 (2012) 2614.

[9] S. de Souza, S.J. Visco, L.C. DeJohnge, J. Electrochem. Soc. 144 (1997) L35.

[10] S.E. Paje, J. Llopis Appl. Phys. A57 (1993) 225.

[11] B. Savoini, J.E. Muñoz Santiuste, R. González, Phys. Rev. 56 (1997) 5856.

[12] N.G. Petrik, D.P. Taylor, T.M. Orlando, J. Appl. Phys. 85 (1999) 6770.

[13] S. Gutzov, J. Mater. Sci. Lett. 19 (2000) 275.

[14] H. Nakajima, T. Mori, S. Itoh, M. Watanabe, Sol. St. Commun. 129 (2004) 421

[15] J. D. Fidelus, S. Yatsunenko, M. Godlewski, W. Paszkowicz, E. Werner-Malentob, W. Łojkowski, Scr. Mater. 61 (2009) 415.

[16] R. Halder, P. Sengupta, V. Sudarsan, A. Ghosh, A. Ghosh, A. Bhukta, G. Sharma, I. Samajdar, G. K. Dey, J. Nucl. Mater. 456 (2015) 359.

[17] Y. Fukuda, S. Moriyama, I. Ogawa, Nucl. Instrum. Methods Phys. Res. Sect. A 732 (2013) 397. 
[18] A. Fukabori, T. Yanagida, J. Pejchal, S. Maeo. Y. Yokota, A. Yoshikawa, T. Ikegami, F. Moretti, K. Kamada, J. Appl. Phys. 107, 073501 (2010).

[19] D.A. Cusano, C.D. Greskovich, F.A. DiBianca, Patent US4421671 A.

[20] T. Yanagida, J. Lumin. in press, doi:10.1016/j.jlumin.2015.01.006 (2015).

[21] T. Yanagida, K. Kamada, Y. Fujimoto, H. Yagi, T. Yanagitani, Opt. Mat. 35 (2013) 2480.

[22] T. Yanagida, Y. Fujimoto, T. Ito, K. Uchiyama, K. Mori, Appl. Phys. Exp., 7 (2014) 062401.

[23] T. Yanagida, Y. Fujimoto, N. Kawaguchi, S. Yanagida, J. Ceram. Soc. Jpn. 121 (2013) 988.

[24] V.R. PaiVerneker, A.N. Petelin, F.J. Crowne, D.C. Nagle, Phys. Rev. 40 (1989) 8555.

[25] C. Lin, C. Zhang, J. Lin J. Phys. Chem. C 111 (2007) 3300.

[26] N.V. Guerassimova, I.A Kamenskikh, V.V. Mikhailin, I.N. Shpinkov, D.A. Spassky, E.E. Lomonova, M.A. Borik, N.I. Markov, V.A. Panov, M.A. Vishnjakova, M. Kirm, G. Zimmerer, Nucl. Instrum. Methods A 486234 (2002).

[27] P.A. Rodnyi, Physical Processes in Inorganic Scintillators (CRC Press, Boca Raton, FL, 1997).

[28] J.-M. Costantini, F. Beuneu, Ma. Fasoli, A. Galli, A. Vedda, M. Martini, J. Phys.: Condens. Matter 23 (2011) 115901.

[29] J.-M. Costantini, F. Beuneu, Ma. Fasoli, A. Galli, A. Vedda, M. Martini, J. Phys.: Condens. Matter 23 (2011) 455901.

[30] Mckeever S. W.S. Thermoluminescence of Solids, Cambridge University Press. 1985. 